\title{
LOCALLY COMPACT GROUPS WHICH HAVE THE WEAKLY COMPACT HOMOMORPHISM PROPERTY
}

\author{
VOLKER RUNDE
}

(Communicated by J. Marshall Ash)

\begin{abstract}
A locally compact group $G$ is WCHP if every weakly compact homomorphism from $L^{1}(G)$ into a Banach algebra has finite-dimensional range, and is WCHP $^{+}$if every extension of $G$ by an abelian group is WCHP. We verify the $\mathrm{WCHP}^{+}$property for certain locally compact groups, including all Moore groups and all connected groups.
\end{abstract}

A locally compact group $G$ is said to have the weakly compact homomorphism property, or, shortly, to be WCHP, if every weakly compact homomorphism from $L^{1}(G)$ into a Banach algebra has finite-dimensional range ([Joh]). If every extension of $G$ by an abelian group is WCHP, $G$ is called WCHP $^{+}$ ([Joh, Definition 4.1]). All abelian and all compact groups are $\mathrm{WCHP}^{+}$, and every extension of a $\mathrm{WCHP}^{+}$group by a $\mathrm{WCHP}^{+}$group is again $\mathrm{WCHP}^{+}$([Joh, Corollary 4.5]). It is possible that every locally compact group is $\mathrm{WCHP}^{+}$.

In this brief note, we use the heredity results for WCHP $^{+}$groups from [Joh] and structure theorems for certain locally compact groups to establish the $\mathrm{WCHP}^{+}$property for these groups.

Theorem. Let $G$ be a locally compact group, and suppose that any of the following holds:

(i) $G \in[F D]^{-} \cap[S I N]$.

(ii) $G \in$ [Moore].

(iii) $G$ is almost connected.

Then $G$ is $\mathrm{WCHP}^{+}$.

For the definitions of $[F D]^{-},[S I N]$ and [Moore], see [Pal], for example.

Proof. Let $G \in[F D]^{-} \cap[S I N]$. By [G-M, Theorem 4.6], we have $G \cong V \times H$, where $V$ is a vector group and $H$ is an extension of a compact group by an abelian one. Hence, applying [Joh, Corollary 4.5] twice yields that $G$ is $\mathrm{WCHP}^{+}$.

Now, let $G \in$ [Moore]. Then $G$ is a finite extension of a Takahashi group ([Rob]). Since Takahashi groups belong to $[F D]^{-} \cap[S I N]$, it follows from (i) and [Joh, Theorem 4.2(i)] that $G$ is $\mathrm{WCHP}^{+}$.

Received by the editors February 14, 1994 and, in revised form, April 13, 1994.

1991 Mathematics Subject Classification. Primary 43A20; Secondary 22D05, 46H99, 47B48.

(c) 1995 American Mathematical Society 
Finally, let $G$ be almost connected. Since $G$ is the extension of a connected group by a compact one, it suffices in view of [Joh, Corollary 4.5] to assume that $G$ is connected. By [M-Z, Theorem in Section 4.6], $G$ is the extension of a connected Lie group by a compact group. Again using [Joh, Corollary 4.5], we may therefore assume that $G$ is a connected Lie group. Now, by [Wil, Theorem 1.2], there are abelian subgroups $H_{1}, \ldots, H_{n}$ of $G$ such that $G=H_{1} \cdots H_{n}$. Hence, as a consequence of [Joh, Theorem 4.2(ii)], $G$ is $\mathrm{WCHP}^{+}$.

Remarks. 1. In view of the remark in [Joh] preceding Corollary 4.5, our result shows that every connected group is even $\mathrm{WCHP}^{++}$, a property formally stronger than $\mathrm{WCHP}^{+}$.

2. In [Joh], B. E. Johnson asks:

Is it possible $[\ldots]$ to show that if every irreducible representation of $L^{1}(G)$ is finite-dimensional, then $G$ is WCHP?

If "irreducible representation" is supposed here to mean "topologically irreducible *-representation on a Hilbert space", as is usual in the group algebra context, this question asks if every $G \in$ [Moore] is WCHP and is thus resolved by our theorem.

\section{REFERENCES}

[G-M] S. Grosser and M. Moskowitz, Compactness conditions in topological groups, J. Reine Angew. Math. 246 (1971), 1-40.

[Joh] B. E. Johnson, Weakly compact homomorphisms from group algebras, Proc. Amer. Math. Soc. 119 (1993), 1249-1258.

[M-Z] D. Montgomery and L. Zippin, Topological transformation groups, Interscience, New York, 1955.

[Pal] T. W. Palmer, Classes of nonabelian, noncompact, locally compact groups, Rocky Mountain J. Math. 8 (1978), 682-741.

[Rob] L. C. Robertson, A note on the structure of Moore groups, Bull. Amer. Math. Soc. 75 (1969), 594-599.

[Wil] G. A. Willis, The continuity of derivations from group algebras: Factorizable and connected groups, J. Austral. Math. Soc. Ser. A 52 (1992), 185-204.

Fachbereich 9 Mathematik, Universität des SaArlandes, Postfach 151150, 66041 SaARBRÜCKEN, GERMANY

E-mail address: runde@math.uni-sb.de 